\title{
Design Optimization of Piezoelectric Energy Harvesting Cantilever for Medical Devices
}

\author{
Mariem Saida, Ghada Zaibi, Mounir Samet and Abdennaceur Kachouri
}

\begin{abstract}
Energy harvesting from the human body is considered as an effective solution for powering biomedical systems. More particularly, the piezoelectric energy recovery from the human body associated mechanical vibrations is discovered to represent a highly promising solution. Relevant research works as perceived, in the related literature, turns out to reveal showed that the harvested power depends on several intervening factors such as the geometry, size and materials used in the construction of the piezoelectric cantilever. In addition, the reduction and the change of the design of the piezoelectric system appear to constitute important factors in output power enhancing process. In this context, the present work is conceived to propose a novel design of the piezoelectric cantilever. Accordingly, a special study dealing with conventional rectangular shape of the piezoelectric energy harvester is conducted. Hence, the major contribution of this study consists in an attempts to provide a newly designed cantilever of a new shape in a bid to enhance the scavenged power for low frequencies. To this end, various structures are tested and compared through implementation of the finite element method (FEM). The proposed associated performance is analyzed in terms of such factors as the von Mises stress, displacement, voltage and output power. The ultimate simulation results prove to indicate that the proposed architectural design turns out to be able to generate an electric power of a range of $14.11 \mu \mathrm{W}$ at a resonant frequency of $8.5 \mathrm{~Hz}$. It is also worth noting that the advanced novel shape is discovered to exhibit the most effective performance levels in respect of some other observed designs subject of investigation.
\end{abstract}

Index Terms-Cantilever, energy harvesting, human body vibration, medical device, new shape, piezoelectric.

\section{INTRODUCTION}

$\mathrm{W}$ ith the growth of medical systems associated miniaturization, the choice of power supply optimal options turns out to stand as a rather too difficult and an critical issue. More particularly, such systems are powered by local batteries that are difficult to replace or recharge. As a means of coping with the energy restriction problems, a considerable interest has been directed to the energy harvesting alternative [1].

Manuscript received May 13, 2019; revised September 4, 2019. Date of publication October 10, 2019. Date of current version October 10, 2019.

Authors are with the Research Laboratory on Electronics and information Technologies: LETI, University of Sfax, National School of Engineering of Sfax, Sfax, Tunisia (e-mails: mariem.saida@enis.tn, ghada_zaibi@yahoo.fr, mounir.samet@enis.rnu.tn, abdennaceur.kachouri@enis.rnu.tn).

Digital Object Identifier (DOI): 10.24138/jcomss.v15i4.770
In effect, the energy harvesting process appears to represent a promising solution whereby batteries can be replaced and to deal with problems related to conventional power supply methods attached problems can be effectively handled such as those relating to the short life time and environmental pollution phenomena. This procedure consists in converting ambient energy (vibration, light, thermal, etc.) into electrical energy to be reused in powering electronic devices [2].

More interestingly, the human body recovered energy represents an effective solution to provide the medical systems with equitable power supply. Actually, the human body constitutes such an intense source of energy [3], that it can produce electrical energy stemming from different sources (movement, walking, breathing, heat, etc.) [4]. Among these sources, the kinetic energy turns out to be accessible in different parts of the human body.

In this context, harvesting energy from the human body related mechanical vibrations are considered as promising energy sources to supply low power electronic devices thanks mainly to the great power density and versatility they can provide.

More particularly, body motions such as the hand, wrist and arm action, walking, joint movement have been considered to stand as attractive sources of mechanical energy whereby biomedical devices can be powered as these diverse movements are characterized with a huge moving distance without the constraint of time [5]. To this regard, various studies have been elaborated in the subject of scavenging electrical energy from the human body excepted motion. Worth citing, in this respect, is Moro and Benasciutti conducted study dealing with a special shoe-mounted to harvest energy from heel accelerations [6]. As for Pillatsch et al, they developed an energy harvester based on human body vibration [7]. In [8], Ylli et al. advanced two inductive energy harvesters: the first is designed to generate energy from the swing motion with the second is conceived to collect electricity from the acceleration pulse upon heel strike. With respect to Delnavaz and Voix, they put forward a special head-mounted to harvest energy from jaw movements [9]. Similarly, Yang et al. developed a special garments enabling to produce electricity from knee and elbow joints.

More recently, three major frameworks have been typically applied to convert vibration into electrical energy namely, the electromagnetic [11], electrostatic [12] and piezoelectric [13] techniques. 
Among them, the piezoelectric conversion proves to be most commonly used mechanism applied to convert vibration into electrical energy thanks to associated advantages of efficiency, simplicity, ease of use and materials variety [14].

In this regard, different piezoelectric structures conversion has been suggested. The most frequently applied among them is the cantilever thanks to the simple structure and high deformation response features it displays [15]. In this report, several attempts and efforts have been made in a bid to enhance the piezoelectric energy harvesting value relevant output power. Among these methods, one can cite the use of different forms of piezoelectric cantilever [16]. In [17], Mehraeen et al demonstrated that the cantilever of tapered shape proves to be rather efficient in producing power as compared to the trapezoidal and rectangular forms culminating in increased energy recovered. Besides, Benasciutti et al, they put forward presented two different trapezoidal cantilever structures enabling to further enhance the harvested power [18]. Similarly, Sameh et al highlighted that a trapezoidal cantilever would generate greater energy amounts relevant to a rectangular shaped cantilever [19]. In [20], Ben Ayed et al investigated a linear and quadratic shape for a cantilever as means for improving the power consumptions scores. The reached results proved to reveal that the quadratic form turns out to generate twice as much power as the conventional rectangular shape. In the same line of thought, the authors in [21] envisaged to design a non-traditional cylindrical shaped cantilever that is attached to a certain number of piezoelectric material made patches.

Hence, an examination of the entirely of the reviewed works reveals well that they have been focused on three major parameters, more specifically, the piezoelectric structure associated dimensions, the materials used as well as the relevant structural to provide the greatest possible amount of energy supplies as collected from the piezoelectric energy harvesters for supplying medical systems. However, there still a great need is still perceived for an effective energy harvesting system to ameliorate the power output.

In this respect, it is necessary to choose the appropriate dimensions and geometry of the piezoelectric structure in order to enhance the harvested power. What makes our work different is that we aim at defining new shape of piezoelectric cantilever which is characterized with a truncated form to ameliorate the harvested energy amount.

In this context, an investigation study of the cantilever related ordinary form is conducted. The aim is to improve the form relevant specifications, thus, improving the relating power recovery capacity. In this regard, a variety of structures are proposed to help reduce the size of the conventional shape. Besides, a great care is dedicated to the cantilever relating different geometric forms whether trapezoidal, triangular and shrunken-triangular. These forms are modeled and simulated by means of the FEM. The achieved simulation results are then compared for the most appropriate shape likely to generate the most optimal energy amount to be selected.

The paper is organized as follows. Section II is devoted to describes the piezoelectric mechanism. Section III deals with depicting the cantilever structure relevant modeling and design features of citing different related shapes and simulation materials. Section IV, it is reserved to highlight the simulation results reached in regard to the rectangular, trapezoidal, and triangular structures, along with those relating to the newly advanced format. A comparison dealing with these different forms associated total displacement, output voltage and output power is established and relevant results reached are then reported. Finally, Section V involves the major concluding the paper.

\section{BACKGROUND}

\section{A. Piezoelectricity Basic features}

Piezoelectricity was initially discovered by the brothers Pierre and Jacques Curie in 1880. It has been defined by two major effect. First, the direct effect which is characterized with the applied material polarization as witnessed under the effect of a mechanical stress. Second, the inverse effect which corresponds to the appearance of a mechanical deformation as noticed under the effect of an electric field applied to the material [22]. On exerting a force on the piezoelectric material, a deformation of the material is manifested causing the generation of an electric potential.

Both of the consecutive equations (1) and (2) respectively depict the piezoelectric system related electrical and mechanical behavior [23]. The two formulas depict the interaction between piezoelectric material in terms of stress, strain, charge density displacement and electric field.

$$
\begin{aligned}
& S=s^{E} T+d_{t} E \\
& D=d_{t} T+\varepsilon^{T} E
\end{aligned}
$$

where

$\mathrm{S}$ is the mechanical strain.

$\mathrm{T}$ denotes the applied mechanical stress.

E represents the electric field.

$D$ designates the electric displacement.

$s^{E}$ is the matrix of elasticity under conditions of constant electric field.

$\mathrm{d}_{\mathrm{t}}$ is the piezoelectric coefficient matrix.

$\varepsilon^{\mathrm{T}}$ denotes the permittivity matrix at constant mechanical strain.

Concerning the mechanical model, the device can be modeled in the form of a resonance system with one degree of freedom (DOF), comprising a rigid mass (M), a damper $(\mathrm{C})$, a spring(K) and the piezoelectric element [24]. An applied external force excites the system and the piezoelectric layers scavenged energy should be immediately measured by means of the external resistive load $\mathrm{R}$, connected to both electrodes. The spring mass model is illustrated on Fig. 1, below.

Actually, the model can be described through these equations [25]:

$$
\begin{aligned}
& M \ddot{Z}(t)+C \dot{Z}(t)+K Z(t)+\theta V(t)=F(t) \\
& \theta \dot{Z}(t)-C_{p} \dot{V}(t)-\frac{V(t)}{R}=0
\end{aligned}
$$




$$
F(t)=-M \ddot{Y}(t)
$$

Where:

$\mathrm{M}$ is the total mass $(\mathrm{kg})$.

$\mathrm{K}$ is the total effective stiffness $(\mathrm{N} / \mathrm{m})$.

$\mathrm{C}$ is the mechanical damping (Ns/m).

$\theta$ is the piezoelectric coupling coefficient $(\mathrm{N} / \mathrm{V})$.

$Z(t)$ is the tip displacement $(\mathrm{m})$.

$\mathrm{F}(\mathrm{t})$ is the applied external force $(\mathrm{N})$.

$\ddot{y}$ : is the vibration acceleration $\left(\mathrm{m} / \mathrm{s}^{2}\right)$.

$\mathrm{V}(\mathrm{t})$ : is the electric load voltage $(\mathrm{V})$.

$\mathrm{C}_{\mathrm{p}}$ is the capacitance of the piezoelectric layer $(\mathrm{F})$.

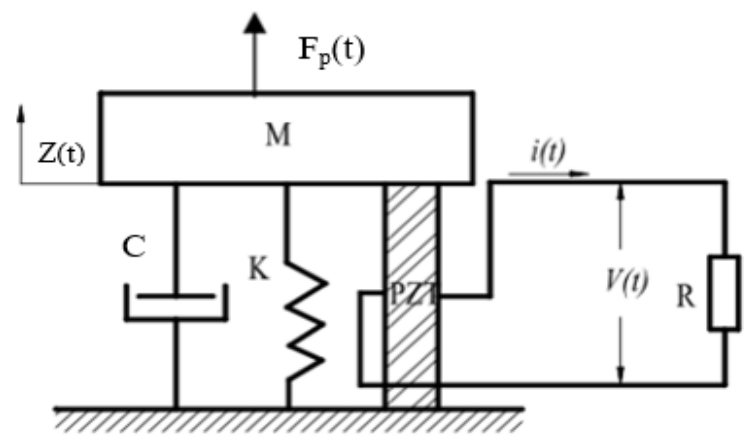

Fig. 1. General mechanical model of piezoelectric system.

In effect, the three equations (3), (4) and (5) can be clearly depicted through an equivalent electric circuit representation [26], as illustrated on Fig. 2.

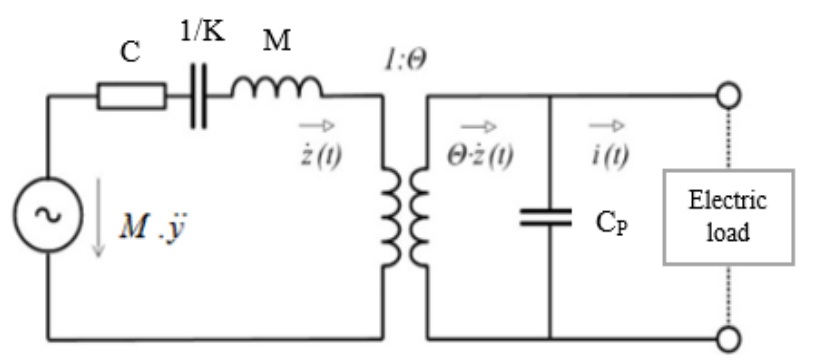

Fig. 2. Equivalent circuit for 1 DOF piezoelectric resonator.

The cantilever structure is the most commonly used mechanism useful for harvesting piezoelectric energy owing mainly to the important responsiveness to low vibrations and easy manufacturing properties it displays as compared to other structures. Additionally, based on the mechanical vibrations, strong constraints can also be generated. More essentially, the resonant frequency of the cantilever associated fundamental mode proves to be very low compared to any other piezoelectric devices associated vibration modes.

According to the relevant literature, various kinds of cantilever structure can be depicted. The most widely applied among them are the unimorph and bimorph cantilever. Commonly, also it is the rectangular shape which turns out to be adopted. Actually, the unimorph type of cantilever is modeled with two layers of various natures, the first is an active and composed of piezoelectric material, while the second layer is inactive and is made of non-piezoelectric material (a metal layer). As far, the bimorph cantilever is composed of an inactive layer inserted between two piezoelectric layers. The general architecture relevant to this cantilever is illustrated on Fig. 3, below.

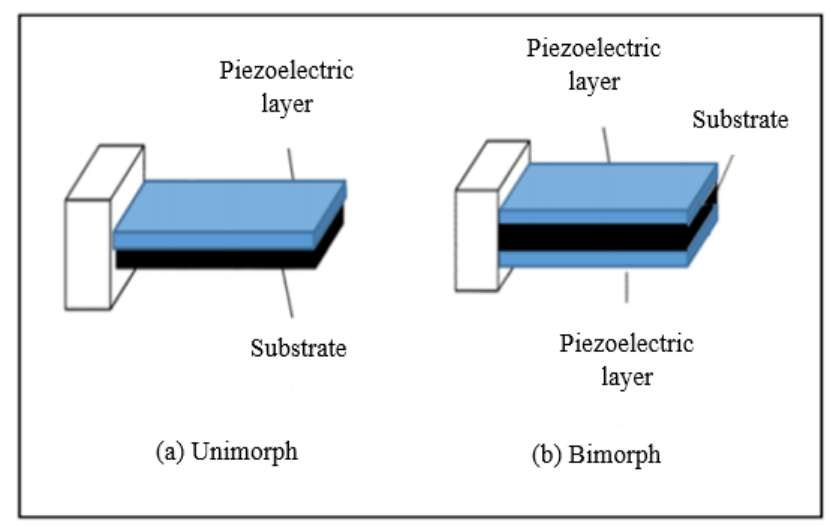

Fig. 3. Structure of the unimorph and bimorph cantilever.

\section{THE ENERGy HARVESTER AND STRUCTURAL MODIFICATION RELATED DESIGNS}

\section{A. The conventional rectangular structure}

\section{1) Materials' selection}

The choice of materials and relevant dimensions has a great impact on the cantilever based power recovery process [18]. In this respect, several types of piezoelectric materials have been suggested in the relevant literature. Among these materials, PZT (Lead zirconate titanate) and PVDF (Polyvinylidene fluoride) prove to be frequently applied for energy recover purposes.

As a polymer, the PVDF displays several advantages as compared to the piezoelectric ceramic PZT. Indeed, it is highly flexible and light weight material. Moreover, the PVDF has small Young's modulus which makes it able to provide the device with a low resonant frequency character [27]. It is, therefore, more adequately fit for maintaining the energy scavenging from low frequency vibration such as the frequency of the human body movements. Actually, the PVDF has been widely used in various human body based energy harvesting applications.

Hence, the fit form usually proposed for the piezoelectric system to take turns out to be the rectangular shape. It is composed of two layers made up of different materials. Indeed, the PVDF material is usually selected to construct the piezoelectric layer while copper, as a material adequately fit for the human body, is chosen usually for the metal layer. The properties of the materials [28], as used for simulation purposes are illustrated on Table I, below.

\section{2) Selecting the cantilever appropriate dimensions}

In this regard, recognizing the source vibration frequency is paramountcy critical, as the piezoelectric proves to produce the highest power range when its resonant frequency appears to correspond to the vibration frequency. It is also worth mentioning that the output power tends to decrease dramatically 
once the source vibration frequency proves to diverge from the resonant frequency [29].

TABLE I

THE Two PVDF AND COPPER MATERIALS ASSOCIATED PROPERTIES

\begin{tabular}{|l|l|l|}
\hline & PVDF & Copper \\
\hline Young's modulus $(\mathrm{GPa})$ & 4 & 110 \\
\hline Poisson ratio & & \\
\hline Density $\left(\mathrm{Kg} / \mathrm{m}^{3}\right)$ & 0.33 & 0.35 \\
\hline Piezoelectric constant $\mathrm{d}_{31}(\mathrm{C} / \mathrm{N})$ & 1780 & 8960 \\
\hline Piezo voltage constant $\mathrm{e}_{31}(\mathrm{Vm} / \mathrm{N})$ & $23 \times 10^{-12}$ & - \\
\hline Relative permittivity $\varepsilon_{\mathrm{r}}$ & $216 \times 10^{-3}$ & - \\
\hline
\end{tabular}

Hence, the natural resonant frequency associated with the piezoelectric system $\omega_{\mathrm{n}}$ is given by the following equation (6):

$$
\omega_{n}=\sqrt{\frac{K}{M}}
$$

For our study case, the mechanical vibration from the human body usually proves to correspond to very low frequencies ranging, more specifically between $1 \mathrm{HZ}$ and $30 \mathrm{~Hz}$ [30]. Hence, for a lower resonant frequency to be specified, several methods have been applied, worth citing among which, is the approach of adding a mass to the end of cantilever, which turns out to be the most is efficiently fit for our case. The desired cantilever turns out to have a structure bearing one fixed end and another free end for vibration purposes. The conventional unimorph cantilever architecture is illustrated through Fig. 4, below.

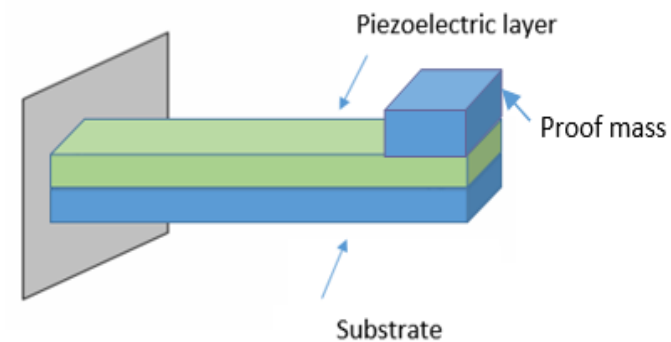

Fig. 4. Structure of the unimorph cantilever with proof mass.

Table II illustrates the different dimensions of a common cantilever can have.

TABLE II

The DifFerent PARAMETERS RELEVANT To THE ReCtangular CANTILEVER

\begin{tabular}{|l|l|l|}
\hline & $\begin{array}{l}\text { Piezoelectric } \\
\text { material } \\
\text { PVDF }\end{array}$ & $\begin{array}{l}\text { Substrate material } \\
\text { Copper }\end{array}$ \\
\hline Length $(\mathrm{mm})$ & 20 & 20 \\
\hline Width $(\mathrm{mm})$ & 10 & 10 \\
\hline Height $(\mathrm{mm})$ & 0.01 & 0.04 \\
\hline
\end{tabular}

\section{B. Application of different forms of cantilever}

The design associated with a unimorph rectangular ordinary cantilever is illustrated through Fig. 5. where L designates the length, $\mathrm{W}$ denotes the width, $\rho_{\mathrm{p}}$ and $\rho_{\mathrm{s}}$ stand respectively for the piezoelectric and non-piezoelectric density, $t_{p}$ and $t_{s}$ represent the piezoelectric and substrate thickness, while $\mathrm{E}_{\mathrm{p}}$ and $\mathrm{E}_{\mathrm{s}}$ denote the Young's modulus relevant to the piezoelectric and substrate layers, respectively.

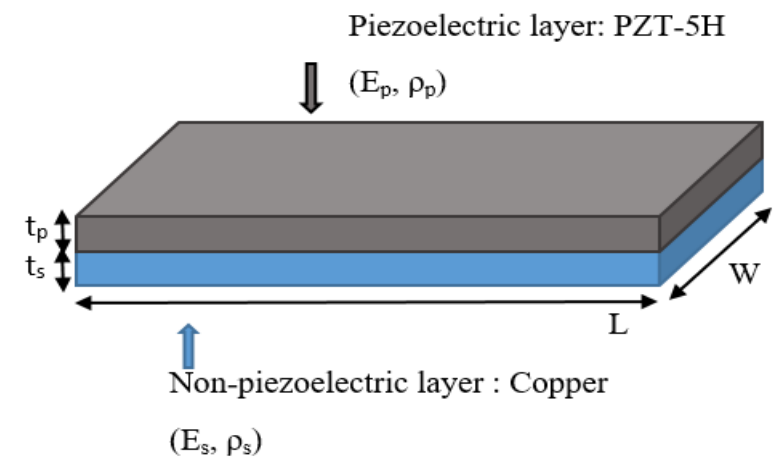

Fig. 5. Geometry of a conventional unimorph cantilever.

The structure related geometry has a considerable impact on the power to recuperate. The major challenge associated with an energy harvester lies in obtaining the maximum possible amount of power. In this regards, several researchers have attempted to increase the size of this recovered power through implementation of various methods.

Based on the above cited scientific research, variation in cantilever related shapes can be considered to stand as an important factor for improving the amount of harvested power, where by the relevant performance can be remarked by improved. Hence, the major objective lying behind conducting the present study consists in constructing optimal form of the cantilever likely to help in maximizing the amount of stored energy. Bearing this, an appropriate structure must be chosen to enhance this power.

By varying the shape of the piezoelectric system, it is necessary to opt for shapes that bear the smallest size possible for the related performance to be noticeably promoted. Based on the conventional form a cantilever can make, we consider attempting to minimize the relevant size, which a way as the relative structure may turn out to be more a singly truncated. As stated in most of the previously conducted research works [31], the more truncated the cantilever structure process to be, the more increased voltage and therefore the output power increases. Accordingly, we consider producing with reducing the relative triangular shape, in order to obtain the new shape proposed, which we dub: shrunken- triangular.

Accordingly, we have designed different shapes: rectangular, trapezoidal, triangular, and the proposed shape and considered to establish a comparison of their respective performances. The entirely of all these configurations associated geometry all shapes are illustrated through Fig. 6, below.

Based on associated dimensions relevant to the rectangular shape of the above figuring cantilever selected above, we reckoned setting different dimensions concerning of the other structures. The different cantilever structural forms under review, along with the relating respective geometrical parameters are depicted through Fig. 7 and on Table III, below. 


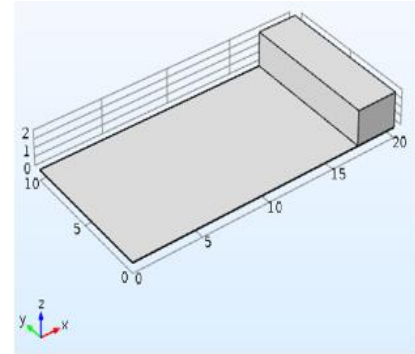

(a)

(c)

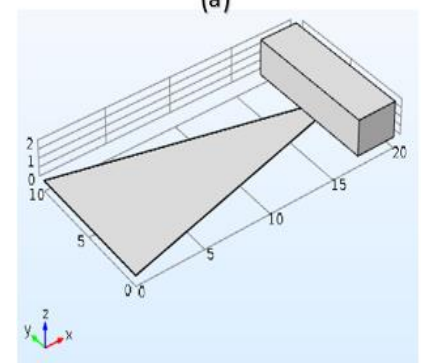

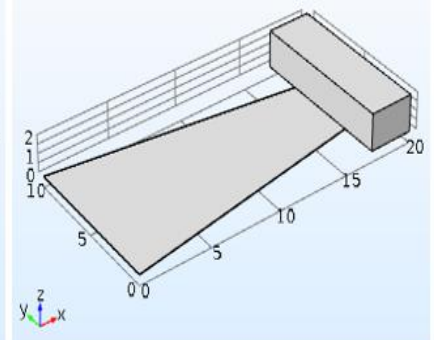

(b)

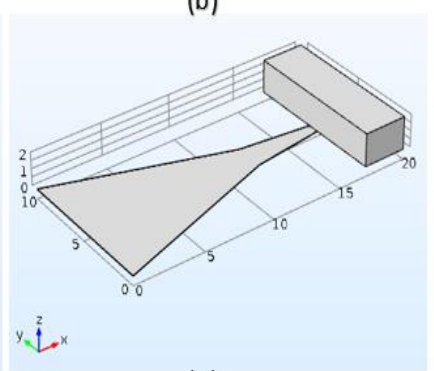

(d)
Fig. 6. Cantilever related shapes: (a) rectangular shaped cantilever (b) trapezoidal shaped cantilever (c) triangular cantilever (d) the proposed shape.

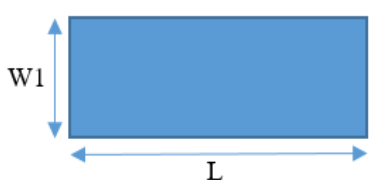

(a)

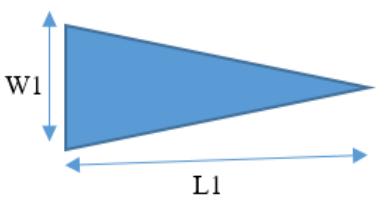

(c)

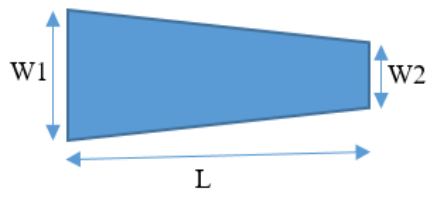

(b)

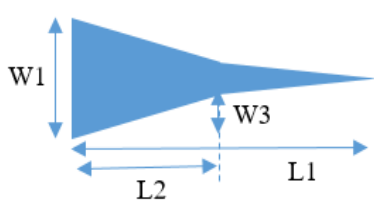

(d)
Fig. 7. Various forms and dimension :(a) rectangular shaped (b) trapezoidal shaped (c) triangular shaped (d) proposed shaped.

TABLE III

GEOMETRICAL PARAMETERS ASSOCIATED WITH THE DIFFERENT SHAPES

\begin{tabular}{lllll}
\hline $\begin{array}{l}\text { Cantilever } \\
\text { form }\end{array}$ & $\begin{array}{l}\text { Length } \\
(\mathrm{mm})\end{array}$ & $\begin{array}{l}\text { Width } \\
(\mathrm{mm})\end{array}$ & $\begin{array}{l}\text { Piezoelectric } \\
\text { thickness }(\mathrm{mm})\end{array}$ & $\begin{array}{l}\text { Substrate } \\
\text { thickness(mm) }\end{array}$ \\
\hline Rectangular & $\mathrm{L}=20$ & $\mathrm{~W} 1=10$ & 0.01 & 0.04 \\
\hline Trapezoidal & $\mathrm{L}=20$ & $\begin{array}{l}\mathrm{W} 1=10 \\
\mathrm{~W} 2=4\end{array}$ & 0.01 & 0.04 \\
\hline Triangular & $\mathrm{L}=20$ & $\mathrm{~W} 1=10$ & 0.01 & 0.04 \\
\hline $\begin{array}{l}\text { Proposed } \\
\text { form }\end{array}$ & $\mathrm{L} 1=20$ & $\mathrm{~W} 1=10$ & 0.01 & 0.04 \\
\hline
\end{tabular}

\section{Analytical modeling of a unimorph piezoelectric} cantilever

Euler Bernoulli's theory is applied to obtain the mathematical modeling of the structure. The equation that defines the motion of a beam with a single piezoelectric layer under the influence of the basic excitation is as follows [32]:

$$
\begin{aligned}
& \frac{\partial^{2} M(x, t)}{\partial x^{2}}+\frac{\partial^{2}}{\partial x^{2}}\left(C_{s} I(x) \frac{\partial^{3} z(x, t)}{\partial x^{2} \partial t}\right)+C_{a} \frac{\partial z(x, t)}{\partial t}+ \\
& m(x) \frac{\partial^{2} z(x, t)}{\partial t^{2}}=-\left[m(x)+M_{t} \delta(x-L)\right] \frac{\partial^{2} z_{b}(x, t)}{\partial t^{2}}
\end{aligned}
$$

where

$\mathrm{M}(\mathrm{x}, \mathrm{t})$ is the internal moment of the beam.

$\mathrm{C}_{\mathrm{s}}$ and $\mathrm{C}^{\mathrm{a}}$ are equivalent strain rate and viscous air damping coefficient.

$z(x, t)$ is the transverse displacement of the neutral axis.

$\mathrm{Z}_{\mathrm{b}}(\mathrm{x}, \mathrm{t})$ is the base excitation displacement.

$\mathrm{I}(\mathrm{x})$ is the area moment of inertia.

$\mathrm{m}(\mathrm{x})$ is the mass per unit length of the beam.

and $\mathrm{M}_{\mathrm{t}}$ is the tip mass.

In this case, the width of the piezoelectric layer is identical to the width of the substrate layer, noted by w. The internal moment $\mathrm{M}(x, t)$ is determined by integrating the stress moment through the thickness, it can be written as:

$$
M(x, t)=-\int_{t_{s}} \sigma_{s} w(x) z d z-\int_{t_{p}} \sigma_{p} w(x) z d z
$$

Where $\sigma_{s}$ is the normal stress in $x$ direction at substrate and $\sigma_{p}$ is the normal stress in $x$ direction at piezoelectric layer. These two terms are defined by:

$\sigma_{s}=E_{s} \varepsilon_{1}^{S}(x, t)$
$\sigma_{p}=E_{p}\left(\varepsilon_{1}^{p}(x, t)-d_{31} E_{3}(t)\right)$

where $E_{p}$ and $E_{s}$ and are the modulus of elasticity of piezoelectric and substrate materials, respectively, $\varepsilon_{1}$ is the mechanical strain, $d_{31}$ is the piezoelectric strain constant Piezoelectric electromechanical coupling is mathematically modeled by $d_{31}, \mathrm{E}_{3}$ is the applied electric field through the $Z$ direction, $\mathrm{E}_{3}$ can be written as a function of the generated voltage $\mathrm{v}(\mathrm{t})$ as follows:

$E_{3}(t)=-v(t) / t_{p}$

The bending strain, $\varepsilon$ at any level z, can be described as below:

$\varepsilon_{1}(x, t)=-z\left(\frac{\partial^{2} z(x, t)}{\partial x^{2}}\right)$

Substituting (3) and (4) into (2) gives the internal bending moment as:

$M(x, t)=E I(x) \frac{\partial^{2} z(x, t)}{\partial x^{2}}+f(x) v(t)$

Where

$f=-\frac{E_{p} w(x) d_{31}}{2}\left(t_{p}+t_{s}\right)$

Where $\mathrm{EI}(\mathrm{x})$ is the flexural rigidity of the composite beam and is calculated as:

$E I(x)=w(x)\left[E_{s} \frac{t_{s}^{3}}{12}+2 \frac{E_{p}}{3}\left(\left(\frac{t_{s}}{2}+t_{p}\right)^{3}-\frac{t_{s}^{3}}{8}\right)\right]$ 
The average strain of piezoelectric cantilever can be determined for various geometries, which is given by:

$\varepsilon(x . t)=-z \frac{\partial^{2} z(x, t)}{\partial x^{2}} \approx-\frac{M(x, t) z}{E I(x)}$

The generated voltage is the integration of the strain function. Consequently, the increase in voltage is related to the increase in the strain function.

$V=-\frac{d_{31} E_{p} t_{p}}{C_{P Q}} \int_{0}^{L} \frac{M(x, t) z}{E I(x)} d x$

Where $\mathrm{Q}$ is the total charge created by the piezoelectric element, which can be defined as:

$Q=-\int_{0}^{L} d_{31} E_{P} t_{p}\left(\frac{M(x, t) z}{E I(x)}\right) d x$

In general, the width function $\mathrm{w}(\mathrm{x})$ of the typical beam geometry is determined by:

$w(x)=$ ratio $\times w(0)+\frac{w(0)(1-\text { ratio })}{l} L-x$

where $w(0)$ is the width of the fixed end and the ratio is the widths ratio of the two extreme sides of the cantilever.

In our case, the proposed shape is the result of the addition of a trapezoidal cantilever and a triangular cantilever of the identical thickness, length L2 and L1 and width w1 and w4 respectively, as illustrated in Fig.8.

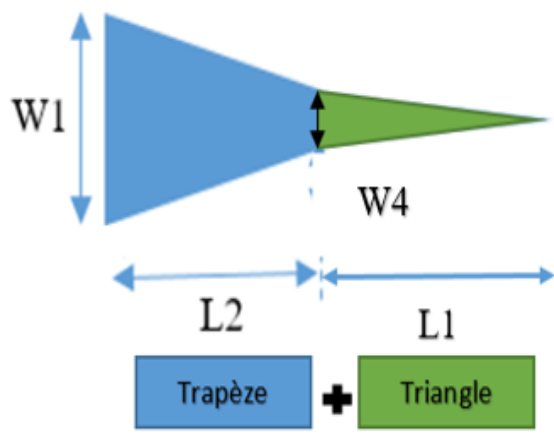

Fig. 8. Dimensions of the proposed form.

Obviously, for the proposed cantilever beam, the width is a function of $\mathrm{x}$ :

Triangle: $w(x)=w_{4}\left(1-\frac{x}{L_{1}}\right)$

Trapeze: $w(x)=\frac{\left(w_{1}-w_{4}\right)\left(L_{2}-x\right)}{L_{2}}+w_{4}$

For the proposed form, $\mathrm{w}$ is as follows:

$w(x)=w_{4}\left(1-\frac{x}{L_{1}}\right)+\frac{\left(w_{1}-w_{4}\right)\left(L_{2}-x\right)}{L_{2}}+w_{4}$
Based on Equation (17), it can be seen that the integral of the strain function must be increased in order to increase the generated voltage. So we must reduce $\mathrm{EI}(\mathrm{x})$ which is reduced by the diminution of the width $\mathrm{w}$. Therefore, the shrunken triangular shape is proposed.

\section{The Simulation REACHEd RESUlts}

This section is devoted to discuss the simulation attained results. In addition to the already detailed mathematical model, we undertake to evaluate the proposed structure via the finite element method to highlight the proposed solution associated performance. This designed piezoelectric system is conceived to be placed on the human body. It can convert vibrations from the human body stemming vibrations into electrical energy. To note, the human body vibration associated frequencies range between $1 \mathrm{~Hz}$ and $30 \mathrm{~Hz}$. The different structures have been studied by imposing an excitation acceleration ( $0.2 \mathrm{~g})$. A copper proof of an $\mathrm{m}=0.53 \mathrm{~g}$, attached proof to the free end of cantilever mass is applied. With respect to our study case, the oscillations reach their maximum value once the human body related vibration frequency proves to correspond to the cantilever associated resonant frequency of the cantilever. As outlined through Fig. 9, the unimorph rectangular cantilever appears to be composed of two different layers and a proof mass.

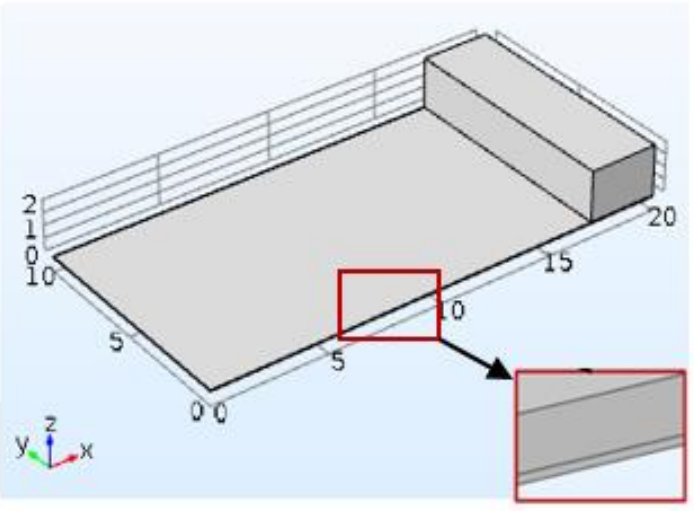

Fig. 9. Design of the proof mass attached cantilever.

We then turn to studying of the characteristics associated with the different investigated forms rectangular, trapezoidal, triangular and the proposed shape, adding mass to the end of each form under review proposed forms. The respective simulation estimation studies are executed by means of the FEM, applied for the purpose of establishing an accurate comparison of the different observed structures with precision, at this level, a number of highly specific criteria are worse considering, namely: the von Mises stress, displacement, voltage, output power and structural volume. Furthermore, the various forms associated characteristics are thoroughly examined based on a specific frequency study.

In a first place, the von Mises stress distribution is implemented on at the resonant frequency determining various structures along the cantilever. The simulation results are depicted on Fig. 10, below. 


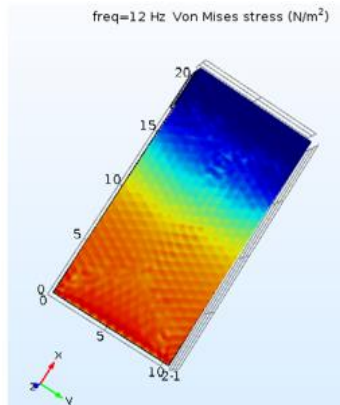

(a)

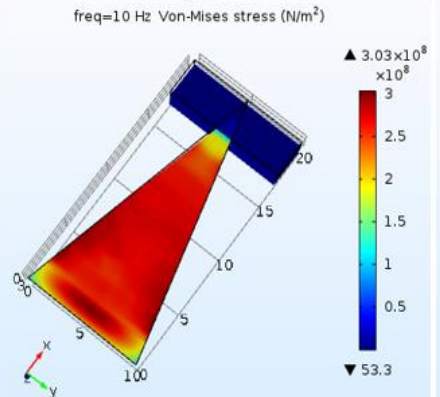

(c)

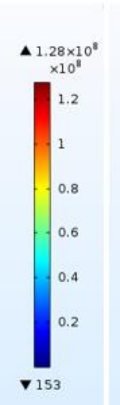

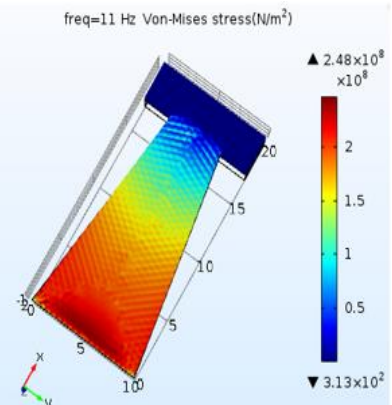

(b)

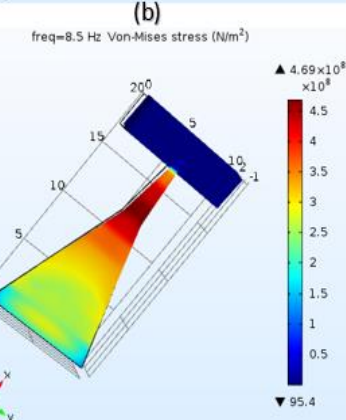

(d)
Fig. 10. The distribution of von Mises stress for various forms of piezoelectric cantilever.

As the figure highlights the maximum von Mises stress as respectively generated for the rectangular, trapezoidal, triangular and the proposed shapes turn out to be: $1.28 \times 10^{8}$ $\mathrm{N} / \mathrm{m}^{2}, 2.48 \times 10^{8} \mathrm{~N} / \mathrm{m}^{2}, 3.03 \times 10^{8} \mathrm{~N} / \mathrm{m}^{2}$ and $4.69 \times 10^{8} \mathrm{~N} / \mathrm{m}^{2}$. Hence, one could well deduce that the proposed architecture turns out to display the largest von Mises stress related distribution.

In a second place, the total displacement of a various cantilever related shapes of cantilever is depicted and simulated via the FEM according to a frequency range comprised between $1 \mathrm{~Hz}$ to $20 \mathrm{~Hz}$, which correspond to the vibrational frequency margins associated with of the human body. The simulation achieved results are depicted on Fig.11, below.

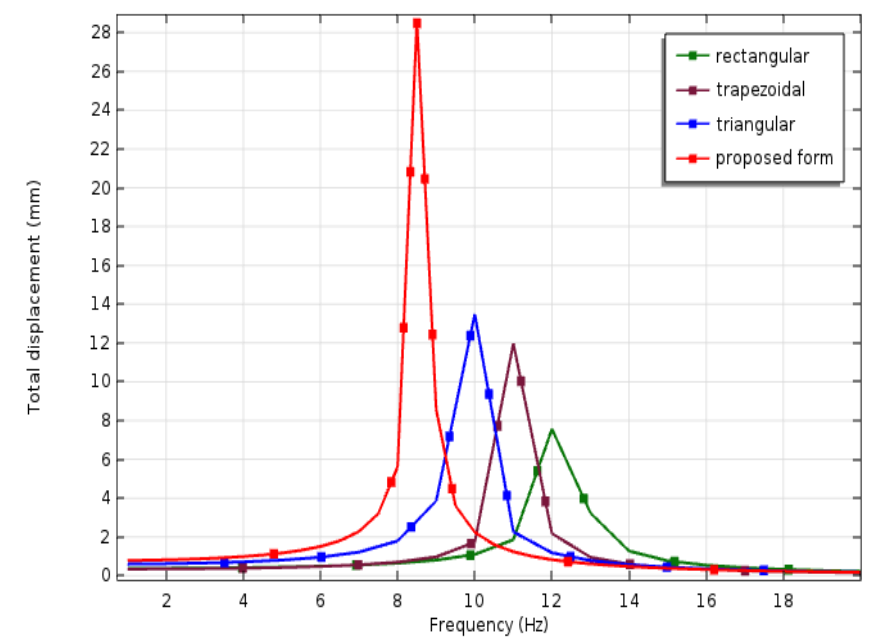

Fig. 11. The displacement of different forms of cantilever.

An examination of the frequency analysis simulation test performed on the different obtained cantilever shapes, achieved reveals well a peak attained at the respective resonant frequencies of $12 \mathrm{~Hz}, 11 \mathrm{~Hz}, 10 \mathrm{~Hz}$ and $8.5 \mathrm{~Hz}$ respectively associated with rectangular, trapezoidal, triangular and proposed forms. More specifically, the proposed shape turns out to score the highest displacement value.

Subsequently, the various shapes respectively generated voltages are extracted. Fig. 12 below outlines the administered simulation reached results of this simulation.

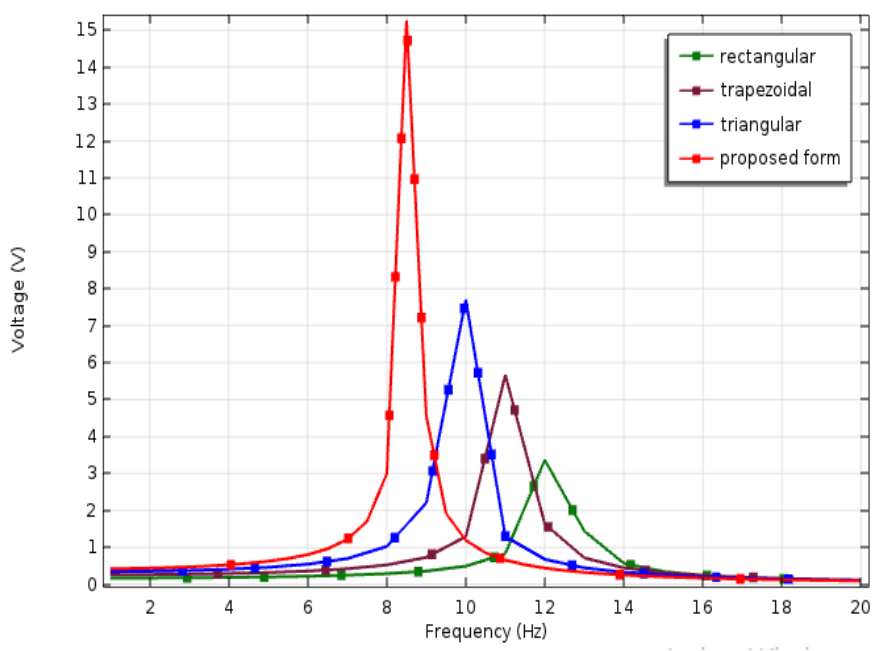

Fig. 12. The voltage value associated with the various cantilever related forms versus frequency.

It can be noted, the rectangular structure turns out to yield a resonant frequency of a range of $3.36 \mathrm{~V}$, i.e the equivalent of 12 $\mathrm{Hz}$, whole the trapezoidal structure proves to yield $5.66 \mathrm{~V}$ at a resonant frequency of $11 \mathrm{~Hz}$, the triangular structure turns out to produce $7.68 \mathrm{~V}$ at a resonant frequency of $10 \mathrm{~Hz}$, whereas the proposed structure proves yield a maximum voltage range of $15.26 \mathrm{~V}$ at a resonant frequency of $8.5 \mathrm{~Hz}$.

Noteworthy also, is the fact that on reducing the form size, the output voltage value proves to increase, while the resonant frequency decreases. Based on such findings, one might well note that the highest voltage value has been reached through implementation of the suggested design.

On the other hand, a comparison is established to the electric power provided by each of the different observed configurations. In this regards, the electrical power output delivered to the resistive load is measured by (23):

$$
P=\frac{V^{2}}{R}
$$

Where:

$\mathrm{R}$ : denotes the resistive load.

$\mathrm{V}$ : stands for the voltage persistence across the resistive load.

The maximum electrical power output is attained once; $\mathrm{R}$ turns out to be equal to $\mathrm{R}_{\text {optimum: }}$ the optimum load resistance, as expressed by the following equation (24) [33]:

$$
R_{\text {optimum }}=\frac{1}{\omega_{r} C_{p}}
$$

Where $\omega_{r}$ denotes the cantilever resonant frequency of cantilever and $C_{p}$ indicates the capacitance of piezoelectric 
device associated capacitance. The later in computed through [33]:

$$
C_{p}=\frac{\varepsilon_{r} \varepsilon_{0} A}{t_{p}}
$$

Where $\varepsilon_{\mathrm{r}}$ designates the relative permittivity, $\varepsilon_{0}$ denotes the absolute permittivity, $t_{p}$ indicates the relevant thickness of piezoelectric layer and $\mathrm{A}$ stands for the area of piezoelectric layer.

Actually, the optimal load resistance associated with the rectangular, trapezoidal, triangular as well as the proposed shape turn out to be: $4.6 \mathrm{M} \Omega, 7.6 \mathrm{M} \Omega, 11 \mathrm{M} \Omega$ and $16.5 \mathrm{M} \Omega$, respectively. Then, we turn to represent the power delivered to the optimal load resistance relevant to each single form. Fig. 13, below, illustrates the simulation achieved results.

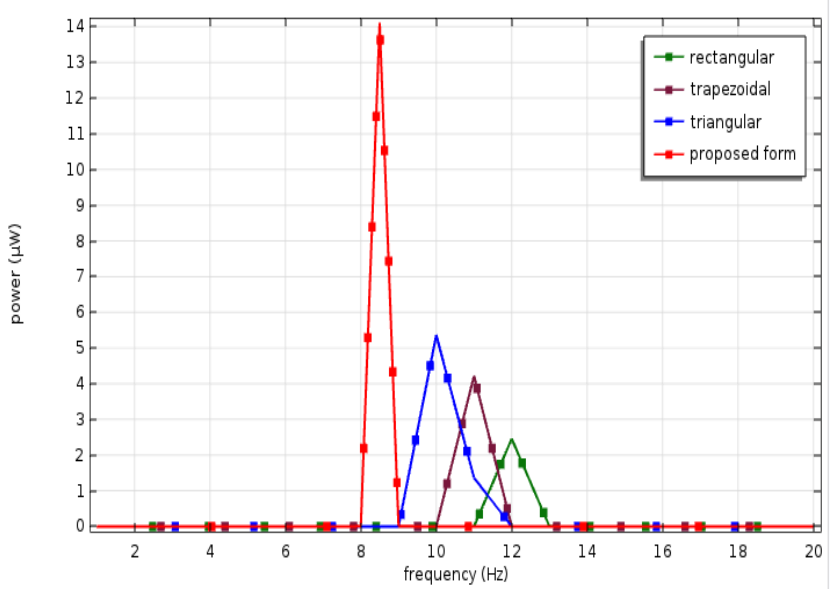

Fig. 13. The power value associated with the various cantilever forms.

The computed power output related to the device with the novel advanced structure bearing device turns out to be equal to $14.11 \mu \mathrm{W}$, as compared to $2,4 \mu \mathrm{W}$ related to the rectangular shaped structure, 4,6 $\mu \mathrm{W}$ regarding the trapezoidal structure and 5,3 $\mu \mathrm{W}$ concerning triangular structure, respectively. Based on the above figuring reached results, it appears clear that the optimized design associated power output which is equal to $14.11 \mu \mathrm{W}$, proves to exceed remarkably those recorded through the entirety of the all other various cantilever shapes under review.

In a next stage, it is interesting to evaluate the volume occupied by each form of cantilever to choose the best performing structure in this respect. Based on the simulation reached achievements, Fig. 14 describes the obtained volume size relevant to each observed form of the cantilever.

As outlined through this figure, the variation the piezoelectric structure associated shape results in the modification of its volume. It is clear by noticeable that the novel structure advanced proves to exhibit the smallest volume range.

It is worth noting, also that by decreasing the free end width of the piezoelectric cantilever, the displacement, the output voltage and the optimal load harvested power at the optimal load turn out to increase, while the resonant frequency is discovered to decrease.
In effect, the shrunken-triangular shaped cantilever appears to display the highest voltage and output power with a range of $15.26 \mathrm{~V}$ and $14.11 \mu \mathrm{W}$, respectively, as noticed at the level of the first resonant frequency. Besides, it proves to exhibit the greatest von Mises stress rate, which turns out to be equal to $4.69 \times 108 \mathrm{~N} / \mathrm{m} 2$.

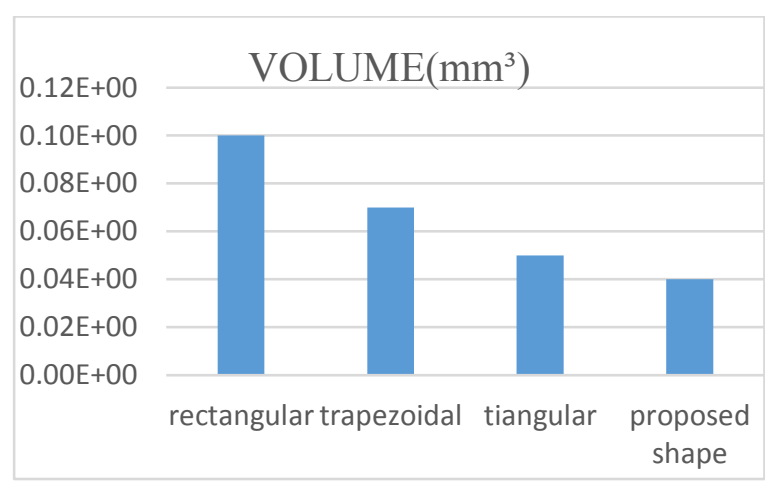

Fig. 14. The different cantilever forms related volumes

The output power, as generated by the proposed form, appears to be equal to $14.11 \mu \mathrm{W}$. It is clearly noticeable that this rate proves to exceed those scored by the other biomechanical harvesters generated output power rates, such as those relating to low frequency $(2.4 \mu \mathrm{W})$ [34].

To sum it up, we could well deduce that for changing in the shape of the piezoelectric cantilever such a procedure proves to bring about a promising influence on the piezoelectric energy harvester associated characteristics. It is clear that, the trapezoidal, triangular, and newly suggested structure do prove to yield more highly efficient results relevant to the regular structure. Still, the most out performing results turn out to be recorded via implementation of the shrunken-triangular shaped cantilever. Besides, the electrical characteristics are discovered to be remarkably improved, as well. Accordingly, the newly shaped framework can be considered to stand as a rather effective solution worth applying with energy harvesting devices at low frequencies, to this end.

\section{CONCLUSION}

In the present work, a novel design of piezoelectric unimorph cantilever useful for harvesting energy from mechanical vibration of human body, is put forward. Accordingly, we modeled the conventional rectangular shaped cantilever of the dimensions are $20 \mathrm{~mm} \times 10 \mathrm{~mm} \times 0.05 \mathrm{~mm}$. In the proposed design, the PVDF was used as piezoelectric material while copper was applied as a substrate material. Added to that, a proof mass was attached to the cantilever free end to decrease the resonant frequency. The simulation results prove to reveal well that the newly proposed architecture turns out to exhibit a maximum power of $14,11 \mu \mathrm{W}$ at a resonant frequency range of $8.5 \mathrm{~Hz}$. Noteworthy, also, is that on other regular state of the art structures, the advanced design is discovered to generate an extra range of power. Hence, one may well conclude that based on the proposed technique conceived on the idea of reshaping the conventional system's overall format, the output power turns out to be remarkably improved. As a proposal for a 
potential work study subject we envisage in introducing a special storage circuit where by medical devices could be rather effectively provisioned with energy on a direct supply bass.

\section{REFERENCES}

[1] A. Z. Kausar, A. W. Reza, M. U. Saleh and H. Ramiah, "Energizing wireless sensor networks by energy harvesting systems: Scopes, challenges and approaches," Renewable and Sustainable Energy Reviews, vol. 38, pp. 973-989,2014. Doi: 10.1016/j.rser.2014.07.035.

[2] G. Zhou, L. Huang, W. Li and Z. Zhu, "Harvesting ambient environmental energy for wireless sensor networks: a survey," Journal of Sensors, 2014 Doi: 10.1155/2014/815467.

[3] K. Fan, Z. Liu, H. Liu, L. Wang, Y. Zhu and B. Yu, "Scavenging energy from human walking through a shoe-mounted piezoelectric harvester," Applied Physics Letters, vol. 110, no.14, pp. 143902, 2017. Doi: 10.1063/1.4979832.

[4] W. Yang, J. Chen, G. Zhu, J. Yang, P. Bai, Y. Su, Q. Jing, X. Cao and Z. L. Wang, "Harvesting energy from the natural vibration of human walking," ACS nano, vol. 7, no. 12, pp. 11317-11324, 2013. Doi: $10.1021 / \mathrm{nn} 405175 \mathrm{z}$

[5] J. Dewei and L. Jing, "Human power-based energy harvesting strategies for mobile electronic devices," Frontiers of Energy and Power Engineering in China, vol. 3, no. 1, pp. 27-46, 2009. Doi: 10.1007/s11708-009-0002-4.

[6] L. Moro and D. Benasciutti, "Harvested power and sensitivity analysis of vibrating shoe-mounted piezoelectric cantilevers," Smart Materials and Structures, vol. 19, no. 11, pp. 115011, 2010. doi: 10.1088/09641726/19/11/115011.

[7] P. Pillatsch, E. M. Yeatman and A. S. Holmes, "A scalable piezoelectric impulse-excited energy harvester for human body excitation," Smart Materials and Structures, vol. 21, no. 11, pp. 115018, 2012. Doi: 10.1088/0964-1726/21/11/115018.

[8] K. Ylli, D. Hoffmann, A. Willmann, P. Becker, B. Folkmer and Y. Manoli, "Energy harvesting from human motion: exploiting swing and shock excitations," Smart Materials and Structures, vol. 24, no. 2, pp. 025029,2015. Doi: 10.1088/0964-1726/24/2/025029.

[9] A. Delnavaz and J. Voix, "Flexible piezoelectric energy harvesting from jaw movements," Smart Materials and Structures, vol. 23, no. 10, pp. 105020, 2014.Doi: 10.1088/0964-1726/23/10/105020.

[10] J. H. Yang, H. S. Cho, S. H. Park, S. H. Song, K. S. Yun and J.H. Lee, "Effect of garment design on piezoelectricity harvesting from joint movement," Smart Materials and Structures, vol. 25, no. 3, pp. 035012, 2016. Doi: 10.1088/0964-1726/25/3/035012.

[11] P. Glynne-Jones, M. J. Tudor, S. P. Beeby and N.M. White, "An electromagnetic, vibration-powered generator for intelligent sensor systems," Sensors and Actuators A: Physical, vol. 110, no.1-3, pp. 344 349, 2004. Doi: 10.1016/j.sna.2003.09.045.

[12] T. Sterken, P. Fiorini, K. Baert, R. A. P. R. Puers, R. A. P. R and G. A. B G. Borghs, "An electret-based electrostatic/spl mu/generator." TRANSDUCERS'03. 12th International Conference on SolidState Sensors, Actuators and Microsystems. Digest of Technical Papers (Cat. No. 03TH8664). Vol. 2. IEEE, 2003. Doi: $10.1002 / 9783527672943 . c h 8$.

[13] C. Wei and X. Jing, X, "A comprehensive review on vibration energy harvesting: Modelling and realization," Renewable and Sustainable Energy Reviews, vol. 74, pp. 1-18, 2017. Doi: 10.1016/j.rser.2017.01.073.

[14] S. Priya, H. C. Song, Y. Zhou, R. Varghese, A. Chopra, S. G. Kim and R. G. Polcawich (2017). "A review on piezoelectric energy harvesting: materials, methods, and circuits," Energy Harvesting and Systems, vol. 4, no. 1, pp. 3-39, 2017. Doi: 10.1515/ehs-2016-0028.
[15] R. S. Bindu, M. P. Kushal and M. Potdar, M, "Study of piezoelectric cantilever energy harvesters," International journal of innovative research and development, pp. 2278-0211, 2014.

[16] F. Goldschmidtboeing and P. Woias, P, "Characterization of different beam shapes for piezoelectric energy harvesting," Journal of micromechanics and microengineering, vol. 18, no. 10, pp. 104013, 2008. Doi: 10.1088/0960-1317/18/10/104013.

[17] S. Mehraeen, S. Jagannathan and K. A. Corzine, "Energy harvesting from vibration with alternate scavenging circuitry and tapered cantilever beam," IEEE Transactions on Industrial Electronics, vol. 57, no. 3, pp. 820-830, 2010. Doi:10.1109/TIE.2009.2037652.

[18] D. Benasciutti, L. Moro, S. Zelenika and E. Brusa, "Vibration energy scavenging via piezoelectric bimorphs of optimized shapes," Microsystem technologies, vol. 16, no. 5, pp. 657-668, 2010. Doi:10.1007/s00542-0091000-5.

[19] S. B. Ayed, F. Najar and A. Abdelkefi, A, "Shape improvement for piezoelectric energy harvesting applications," International Conference on Signals, Circuits and Systems (SCS 03), IEEE, Nov. 2009, pp. 1-6. Doi :10.1109/ICSCS.2009.5412553.

[20] S. Ben Ayed, A. Abdelkefi, F. Najar and M. R. Hajj, "Design and performance of variable-shaped piezoelectric energy harvesters," Journal of Intelligent Material Systems and Structures, vol. 25, no. 2, pp. 174-186, 2014. Doi: 10.1177/1045389X13489365.

[21] M. S. Nguyen, S.H. Ng, P. Kim and Y. J. Yoon, "Piezoelectric Cylindrical Design for Harvesting Energy in Multi-Directional Vibration Source," IOP Conference Series: Earth and Environmental Science, IOP Publishing, Aug. 2017, Vol. 83, no. 1, pp. 012010. Doi: 10.1088/17551315/83/1/012010.

[22] H. S. Kim, J. H Kim and J. Kim, J, "A review of piezoelectric energy harvesting based on vibration," International journal of precision engineering and manufacturing, vol. 12, no. 6, pp. 1129-1141, 2011. Doi: 10.1007/s12541-011-0151-3

[23] A. Nechibvute, A. Chawanda and P. Luhanga, P, "Piezoelectric energy harvesting devices: an alternative energy source for wireless sensors," Smart Materials Research, 2012. Doi: 10.1155/2012/853481.

[24] E. Lefeuvre, A. Badel, C. Richard, L. Petit and D. Guyomar, "A comparison between several vibration-powered piezoelectric generators for standalone systems," Sensors and Actuators A: Physical, vol. 126, no. 2, pp. 405-416, 2006.Doi: 10.1016/j.sna.2005.10.043.

[25] Y. C. Shu and I. C. Lien, "Analysis of power output for piezoelectric energy harvesting systems," Smart Mater. Struct., vol. 15, no. 6, pp. 1499-1512, Dec. 2006. Doi: 10.1088/0964-1726/15/6/001.

[26] J. Dicken, P. D. Mitcheson, I. Stoianov, and E. M. Yeatman, "PowerExtraction Circuits for Piezoelectric Energy Harvesters in Miniature and Low-Power Applications," IEEE Trans. Power Electron., vol. 27, no. 11, pp. 4514-4529, Nov. 2012. Doi: 10.1109/TPEL.2012.2192291.

[27] T. Starner and J. A. Paradiso, "Human generated power for mobile electronics," Low-power electronics design, vol. 45, pp. 1-35, 2004.Doi: 10.1201/9781420039559.ch45.

[28] C. Akitegetse, C. Volat and M. Farzaneh, "Measuring bending stress on an ice/aluminium composite beam interface using an embedded piezoelectric PVDF (polyvinylidene-fluoride) film sensor, " Measurement Science and Technology, vol. 19, no 6, pp. 065703, 2008.

[29] D. Shen, J. H. Park, J. Ajitsaria, S. Y. Choe,H. C Wikle III and D. J. Kim, "The design, fabrication and evaluation of a MEMS PZT cantilever with an integrated Si proof mass for vibration energy harvesting," Journal of Micromechanics and Microengineering, vol. 18, no. 5, pp. 055017, 2008. Doi: 10.1088/0960-1317/18/5/055017.

[30] D. Zhu, "Vibration energy harvesting: machinery vibration, human movement and flow induced vibration," Sustainable Energy Harvesting Technologies-Past, Present and Future. IntechOpen, 2011. Doi: $10.5772 / 25731$. 
[31] S. Saadon and O. Sidek, "Shape optimization of cantilever-based MEMS piezoelectric energy harvester for low frequency applications," 2013 UKSim 15th International Conference on Computer Modelling and Simulation. IEEE, 2013. Doi: 10.1109/UKSim.2013.125.

[32] A. G. Muthalif, A. G and N. D. Nordin. "Optimal piezoelectric beam shape for single and broadband vibration energy harvesting: Modeling, simulation and experimental results,". Mechanical Systems and Signal Processing, vol.54,pp.417-426,2005. Doi: 10.1016/j.ymssp.2014.07.014.

[33] A. Ceponis, D. Mazika and V. Bakanuskas, "Trapezoidal cantilevers with Irregular cross-sections for energy harvesting systems," Applied Sciences, 2017, vol. 7, no 2, pp. 134. Doi: 10.3390/app7020134.

[34] S. Saadon and O. Sidek, "Comparative study on standard geometrical structures of cantilever-based MEMS piezoelectric energy harvester over T-shaped cantilever beam for low frequency ambient vibrations," J. Optoelectron. Adv. Mater, vol. 16, pp. 612-618, 2014.

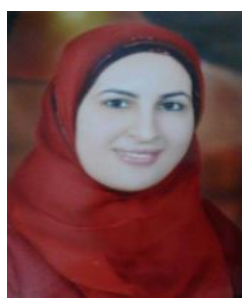

Mariem Saida was born in Sfax, Tunisia, in 1987.She received her Electronic Engineer Diploma in 2011, a Master's degree in electronic in 2013 from the National School of Engineering of Sfax (ENIS). Currently, she is a $\mathrm{PhD}$ student at the Department of Electrical Engineering at National School of Engineering Sfax. Her research interests include energy harvesting, piezoelectric cantilever.

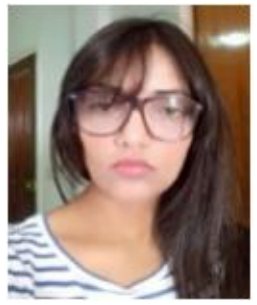

Ghada Zaibi was born in Sfax, Tunisia, in 1982. She received her Engineering Diploma in Electrical Engineering and Master's Degree in Microelectronics from the National School of Engineering of Sfax (ENIS) in 2006 and 2007, respectively. In 2012, she received her $\mathrm{PhD}$ in Electronics and Embedded Systems from University of Sfax, Tunisia and Toulouse le Mirail University, France. She was a contractual assistant professor, from 2007 to 2011, at ENIS, Sfax. She does research in Acoustic Engineering, Communication Engineering, and WSN Security. Her current research focuses on antenna design, energy harvesting, and IOT. Since 2011, she is a permanent Assistant Professor at the National School of Engineering of Monastir (ENIM), University of Monastir.

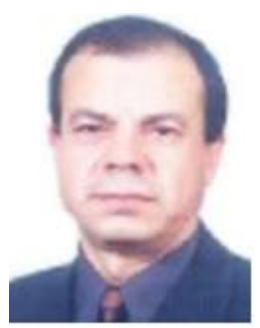

Mounir Samet was born in Sfax, Tunisia, in 1955. He obtained an Engineering Diploma from National School of Engineering of Sfax in 1981, a Master's Degree in Measurement and Instrumentation from National School of Bordeaux (ENSERB) of France in 1981, a Doctorate in Measurement and Instrumentation from ENSERB in 1981, and the Habilitation Degree (Post Doctorate degree) in 1998. $\mathrm{He}$ "works" on several cooperation with medical research groups in Tunisia and France. Currently, he is permanent Professor at ENIS School of Engineering and member in the "LETI" Laboratory ENIS Sfax.

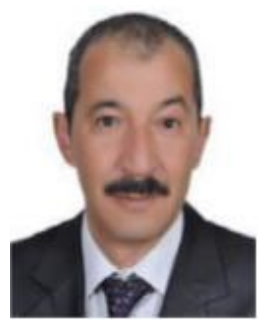

Abdennaceur Kachouri was born in Sfax, Tunisia, in 1954. He received the Engineering Diploma from National School of Engineering of Sfax in 1981, a Master's Degree in Measurement and Instrumentation from National School of Bordeaux (ENSERB) of France in 1981, and a Doctorate in Measurement and Instrumentation from ENSERB in 1983. He "works" on several cooperation with communication research groups in Tunisia and France. Currently, he is permanent Professor at ENIS School of Engineering and member in the "LETI" Laboratory ENIS Sfax. 